\title{
Image Contrast in Energy-Filtered BSE Images at Ultra-Low Accelerating Voltages
}

\section{Yoichiro Hashimoto, ${ }^{1,2,3 *}$ Atsushi Muto, ${ }^{2}$ Eric Woods, ${ }^{4}$ Todd Walters, ${ }^{4}$ and David C. Joy ${ }^{3,5}$}

${ }^{1}$ Hitachi High Technologies Corp., 24-14, Nishi-Shimbashi 1-chome, Minato-ku, Tokyo 105-0003, Japan

${ }^{2}$ Hitachi High Technologies America, Inc., 10 North Martingale Road, Suite 500, Schaumburg, IL 60173

${ }^{3}$ University of Tennessee, Knoxville, TN 37996-2100

${ }^{4}$ Georgia Institute of Technology, 345 Ferst Drive NW, Atlanta, GA 30332

${ }^{5}$ Oak Ridge National Laboratory, 1 Bethel Valley Road, Oak Ridge, TN 37831

*yoichiro.hashimoto.rg@hitachi-hightech.com

\begin{abstract}
Scanning electron microscopy (SEM) at ultra-low landing energies reveals information at the topmost layer of the specimen surface, but the backscattered electron image contrast for certain specimens can be unusual. For primary electron energies above $1 \mathrm{keV}$, backscattered electron (BSE) yields from a specimen increase with increasing atomic number, providing a brighter image for heavier elements. However, at an electron beam energy of $0.2 \mathrm{keV}$, a reversal occurs; the BSE yield is greater for light elements than for heavier elements. The effect has been demonstrated for specimens of $\mathrm{Au}$ and $\mathrm{Si}$ in an SEM with an energyfiltering BSE detector.
\end{abstract}

\section{Introduction}

Morphological and compositional information at the topmost surface of a specimen is important in the field of materials science and engineering because these parameters strongly affect material properties. The scanning electron microscope (SEM) has been one of the best tools to evaluate the surface structure of materials. At higher electron energies the focused beam spot size becomes smaller, allowing better image resolution; thus SEM images are conventionally recorded at electron accelerating voltages from 10 to $30 \mathrm{kV}$. High accelerating voltages, however, are unsuitable for obtaining information from the topmost surface because primary electrons then penetrate deep inside the specimen, enlarging the specimen-electron interaction volume and diluting the information related to the topmost surface. Imaging at low accelerating voltages, with a beam of ultra-low landing energies (ULE), defined as a landing energy below $1 \mathrm{keV}$, is better suited to obtain topmost surface information because the interaction volume and the sampling depth of primary electrons within the specimen becomes small (Figure 1).

Historically, there have been difficulties with SEM imaging at ULE. One specific problem has been chromatic-aberration enlargement of the electron beam spot size, which causes a degradation of image resolution. To overcome this difficulty in the Hitachi SU8200 series SEM, a beam deceleration technique has been applied along with improvements in the electron source, lenses, and detectors. Specifically, a negative voltage is applied to the specimen to decelerate the primary electrons after the final lens and just before the electron beam interacts with the specimen. As a result, this technique reduces beam aberrations and achieves a small spot size at ULE. High-quality images can be obtained even at landing energies less than $100 \mathrm{eV}$. Deceleration also reduces sample beam damage and electron dose.

It is sometimes difficult to interpret SEM data at ULE because the signal behavior at ULE does not follow the conventional theory used at the landing energies above $1 \mathrm{keV}$ [1]. Previous studies provide secondary electron (SE) and backscattered electron (BSE) yields as a function of specimen atomic number and of the incident beam energy; these reference data help to interpret the material contrast at ULE [2]. However, the material contrast obtained by an actual instrument does not always follow these earlier results because of the acceptance capability of detectors and the complex phenomena occurring in the specimen chamber: electron scattering at the chamber wall, generating SE3 electrons. In this article, we describe an investigation of material contrast at ULE in an installed SEM and compare the yields obtained to some reference studies.

\section{Materials and Methods}

Instrument. Figure $2 \mathrm{a}$ shows a general view of the Hitachi SU8230 FE-SEM. The electron source is a cold field emission (CFE) electron gun, which is the best choice for obtaining a small beam spot size at low beam energies. In addition, the CFE

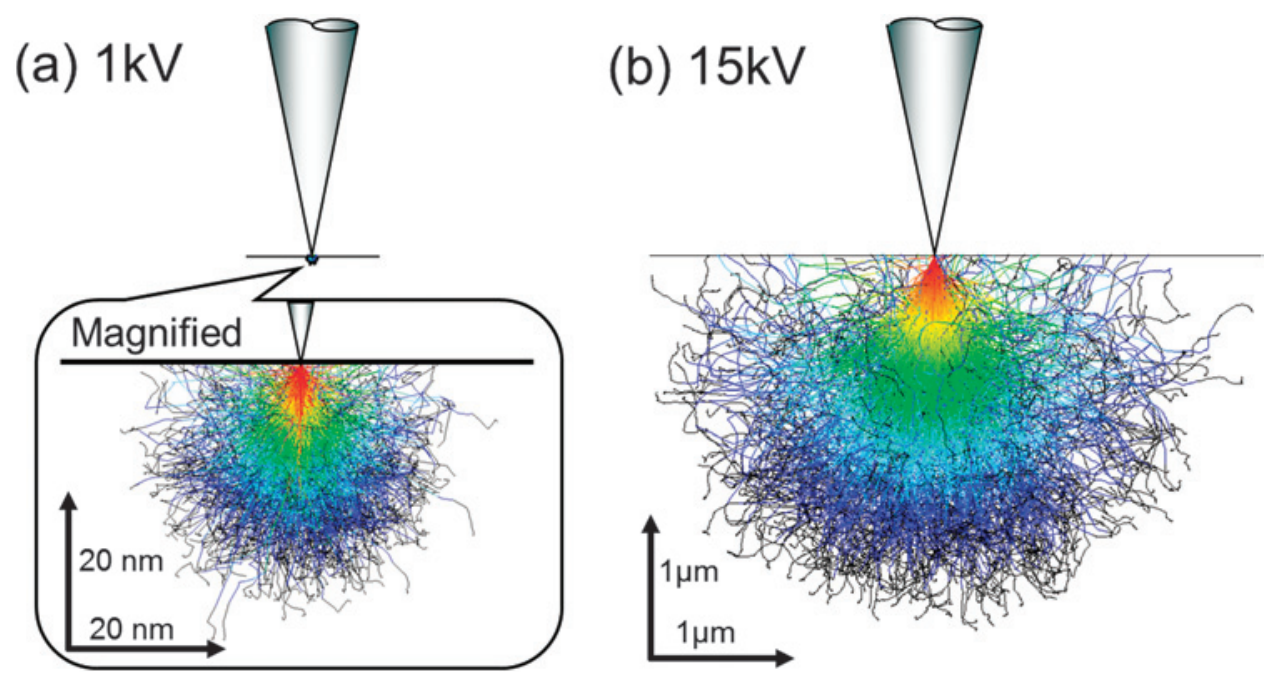

Figure 1: Monte Carlo simulations of electron scattering of primary beam electrons in the specimen (material=carbon). (a) Accelerating voltage $1 \mathrm{kV}$, (b) accelerating voltage $15 \mathrm{kV}$. 


\section{Incomparable...}

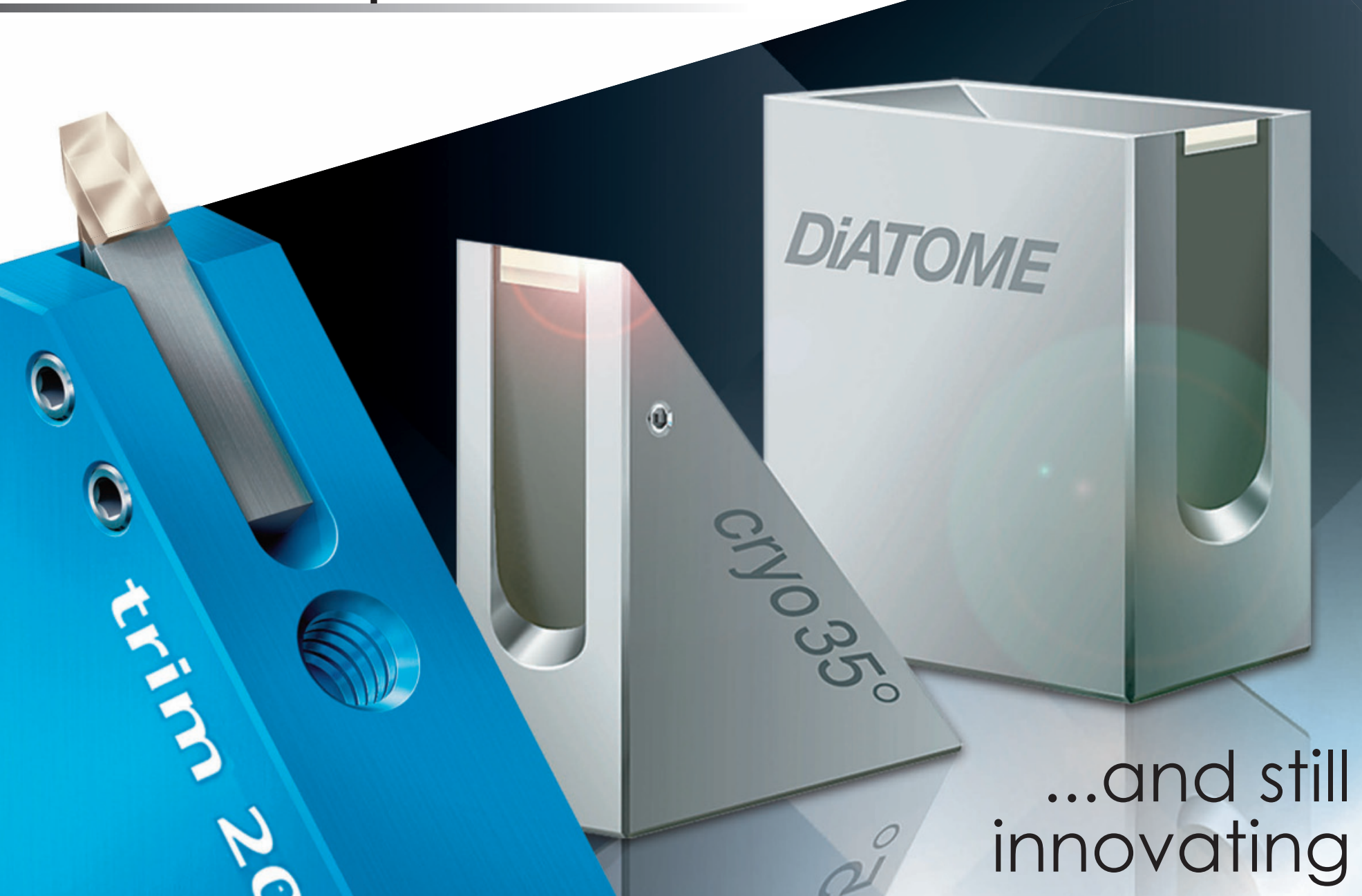

\section{DIATOME diamond knives}

ultra $45^{\circ} \cdot$ cryo $\cdot$ histo ultra $35^{\circ} \cdot$ histo jumbo cryo immuno • ultra sonic ultra AFM \& cryo AFM trimtool $20 \cdot$ trimtool 45 trimtool 90

Over 40 years of development, manufacturing, and customer service

\section{DIATOME U.S.}

P.0. Box 550 • 1560 Industry Rd. • Hatfield, Pa 19440 Tel: (215) 412-8390 • Fax: (215) 412-8450 email: sgkcck@aol.com•www.emsdiasum.com

Free customer service

Sectioning tests with biological and material research specimens of all kinds. We send you the sections along with the surfaced sample, a report on the results obtained and a recommendation of a suitable knife. Complete discretion when working with proprietary samples.

Re-sharpening and reworking service

A re-sharpened Diatome diamond knife demonstrates the same high quality as a new knife. Even knives purchased in previous years can continue to be re-sharpened. The knives can be reworked into another type of knife for no extra charge, e.g. ultra to cryo or $45^{\circ}$ to $35^{\circ}$.

\section{Exchange service}

Whenever you exchange a knife we offer you a new DiATOME knife at an advantageous price.

\section{trimtool 90}

Many requests from customers doing FIB cutting of biological and technical sample blocks have motivated us to relaunch the trim 90 blade:

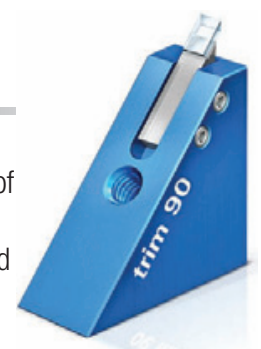

Please contact us for more information.

\section{DiATOME manip}

Our new tool for an easy handling and mounting of section ribbons!
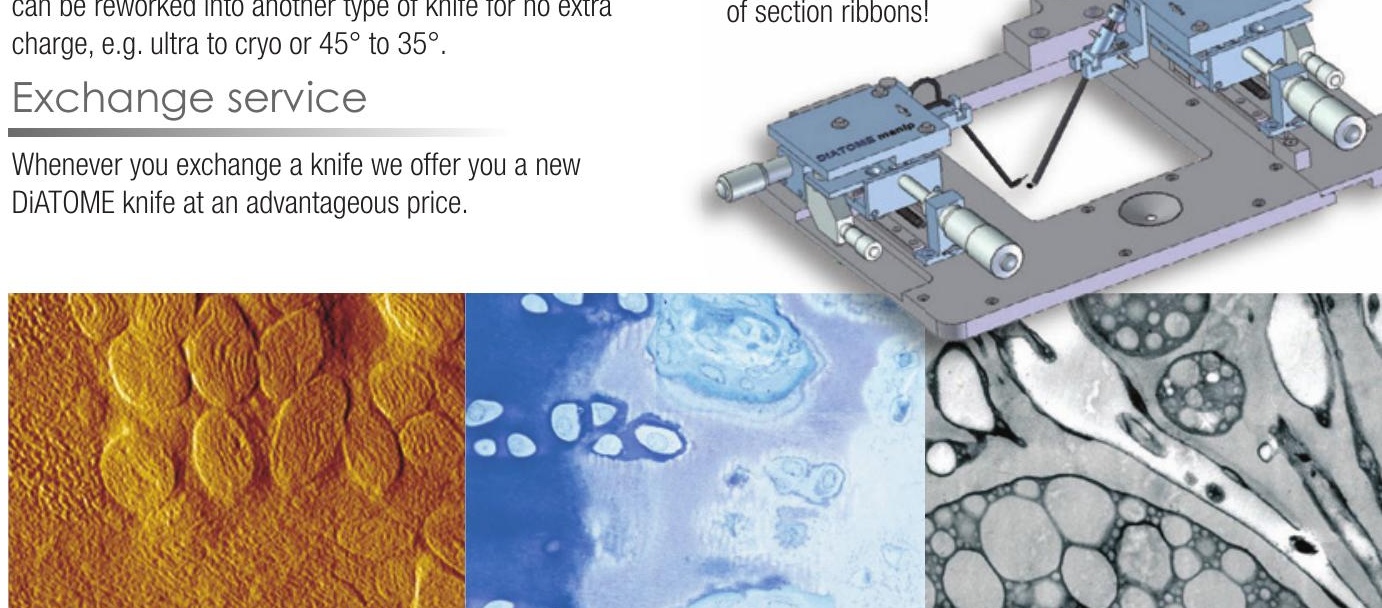

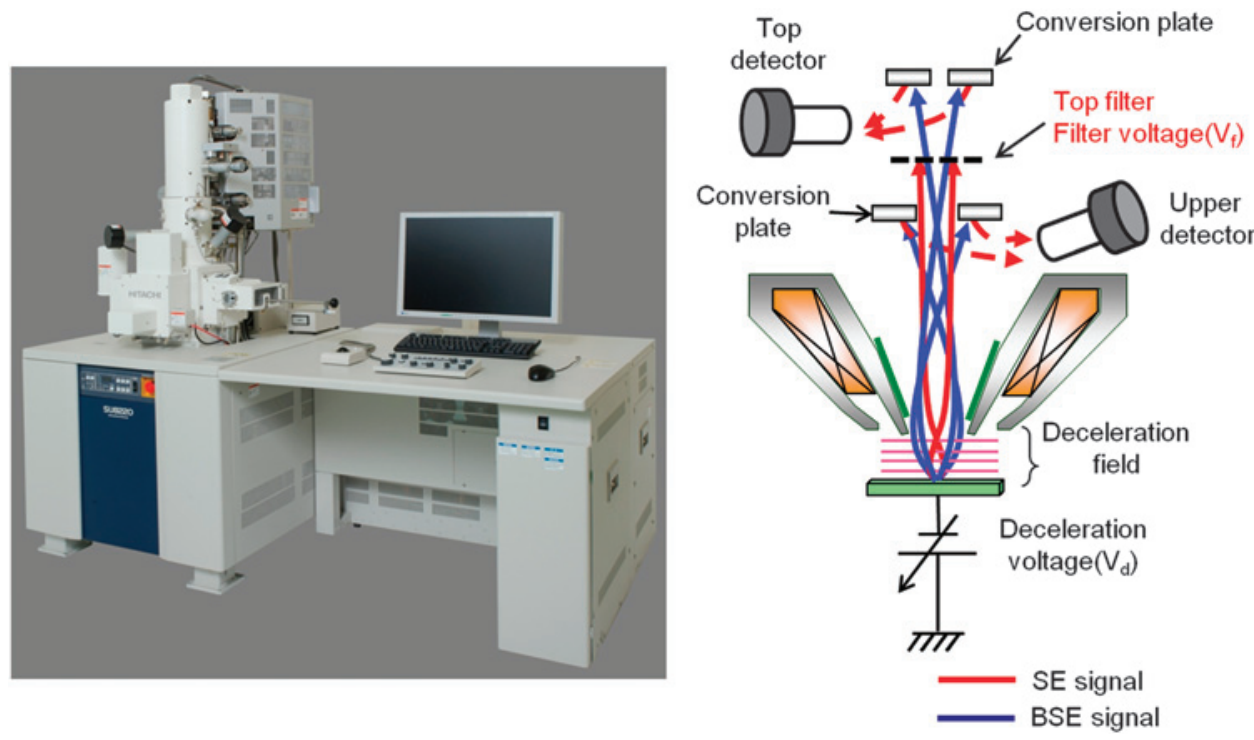

Figure 2: General view of the Hitachi SU8230 SEM (left) and schematic of signal detection system using the deceleration mode (right).
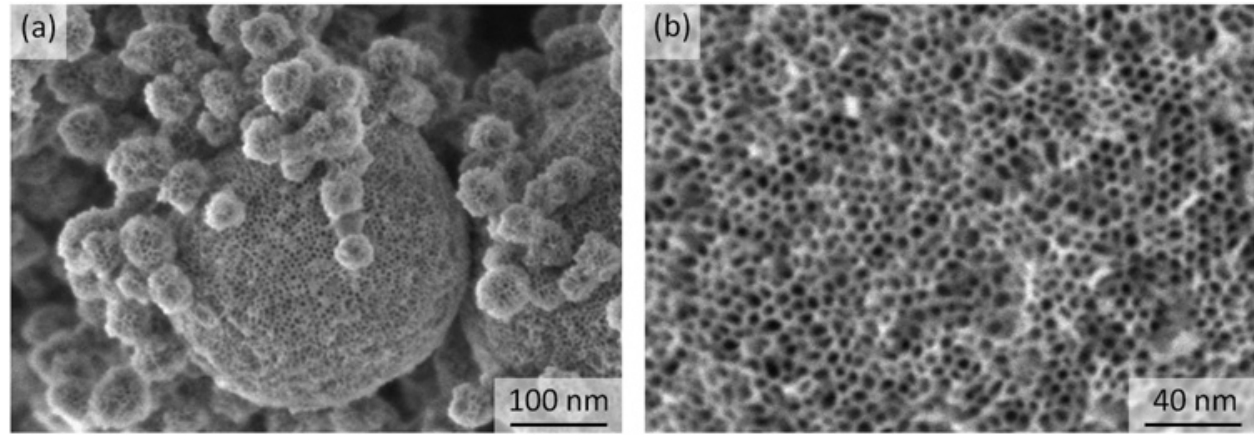

Figure 3: SE + BSE SEM images of mesoporous silica particles acquired at the following conditions: accelerating voltage $=0.5 \mathrm{kV}$. Original magnifications (a) 200,000x and (b) 500,000x. Specimen courtesy of Dr. Toshiyuki Yokoi of Tokyo Institute of Technology.

source has a small energy spread, which reduces the chromatic aberration. The objective lens in the system is a semi-inlens type working at a short focal length, which also reduces aberrations. The system configuration can accommodate large specimens - up to $150 \mathrm{~mm}$ substrates. The standard vacuum of the specimen chamber is on the order of $10^{-5} \mathrm{~Pa}$.

The deceleration function is a standard feature of the Hitachi SU8200 series, allowing high-resolution imaging at accelerating voltages less than $1 \mathrm{kV}$. Figure 3 shows SEM images of mesoporous silica nano particles that are used as catalyst supports or adsorbents. These particles are of amorphous silica, so they are generally susceptible to radiation damage. Therefore, it is necessary to use ULE conditions to reduce sample damage and observe fine structure. In Figure 3 nanopore features about $5 \mathrm{~nm}$ in diameter were observed at $500 \mathrm{eV}$ landing energy with no beam damage and no charging.

The SU8200 series SEM has three types of SE detectorsthe top detector, the upper detector, and the lower Everhart-Thornley detector-which together provide valuable flexibility for imaging. Figure $2 \mathrm{~b}$ shows a schematic of the signal detection system in the deceleration mode. Electrons with high energy and also a low elevation angle will be detected observed with the upper detector. In Figure $4 \mathrm{~b}$, taken with the top detector, the image contrast between CNTs and the PTFE is greater.

Specimens. For this article the BSE yield $\left(\eta_{\exp }\right)$ was measured at low accelerating voltages and compared with reference data from the literature. These measurements were made on carbon, silicon, copper, and gold found on the standards block of an electron probe micro analyzer (EPMA). These specimens were suitable for this experiment because of their purity and their flat surface morphologies. The carbon coating film on the standards block was removed by mechanical polishing before it was provided because it could affect the penetration of primary electrons, especially at ULE, and the escape of BSEs and SEs from the surface.

Methods. The backscattered yield was measured on the standard materials at landing energies from $0.2 \mathrm{keV}$ to $1 \mathrm{keV}$. To compare with reference BSE yields $\left(\eta_{\text {ref }}\right)$ listed in reference [2], the experimental BSE yields $\left(\eta_{\text {exp }}\right)$ were calculated by the following process. The relationship between experimental signal-to-noise ratio $\left(S / N_{\text {exp }}\right)$ and theoretical signal-to-noise ratio $\left(S / N_{\text {theory }}\right)$ is calculated as:

$$
\left(S / N_{\text {exp }}\right)^{2}=k\left(S / N_{\text {Theory }}\right)^{2},
$$


where $k$ is the detective efficiency estimated using electron trajectory simulations and the SE yield of the conversion plate material [3]. $S / N_{\text {exp }}$ can be obtained from:

$$
S / N_{\text {exp }}=\frac{I_{\text {mean }}-I_{D C}}{S_{\text {div }}}
$$

where $I_{\text {mean }}$ is the mean level, $I_{D C}$ is a background level which describes a level when the beam is turned off, and $S_{d i v}$ is a standard deviation of beam intensity. These parameters were obtained from a histogram of actual SEM images. $S / N_{\text {theory }}$ is the signal-to-noise ratio when all the signal is detected, and it can be converted to the number of electrons per pixel $\left(N_{e}\right)$ by:

$$
S / N_{\text {Theory }}=\frac{N_{e}}{\sqrt{N_{e}}}=\sqrt{N_{e}} .
$$
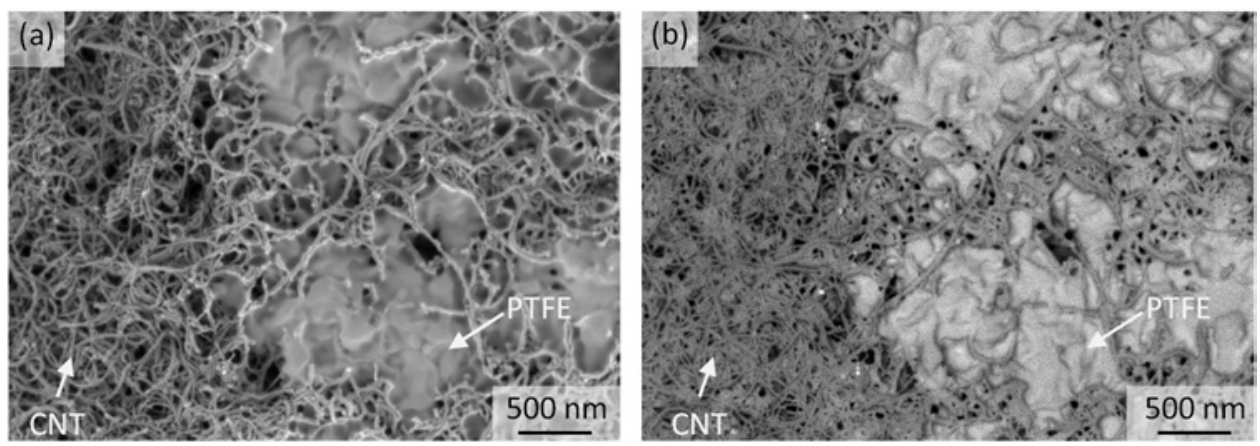

Figure 4: BSE images of CNT/PTFE composite film acquired at the following conditions: accelerating voltage $=0.3 \mathrm{kV}$, deceleration voltage $=0.5 \mathrm{kV}$, and filter voltage $0.7 \mathrm{kV}$. (a) Imaged with upper detector and (b) imaged with top detector. Specimen courtesy of Prof. Yoshiyuki Show of Tokai University.
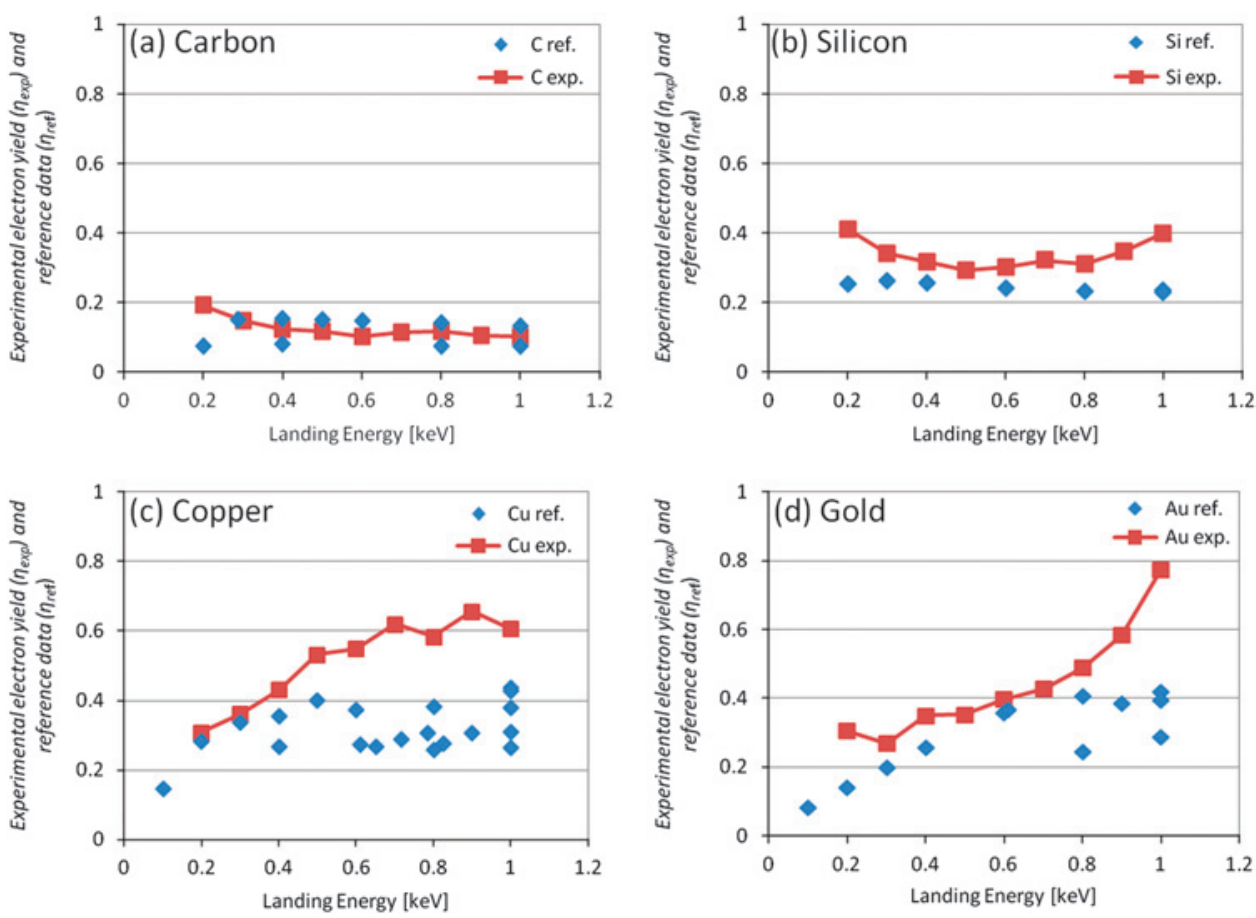

Figure 5: Experimental BSE yields ( $\left.\eta_{\text {exp }}\right)$ versus landing energy compared with reference data $\left(\eta_{\text {ref }}\right)$ for four elements: (a) carbon, (b) silicon, (c) copper, and (d) gold.
By applying the formula (2) and (3) to the formula (1), $N_{e}$ can be calculated by:

$$
N_{e}=\frac{1}{k}\left(\frac{I_{m e a n}-I_{D C}}{S_{d i v}}\right)^{2}
$$

The $\eta_{\exp }$ values were calculated by:

$$
\eta_{\text {exp }}=\frac{e N_{e}}{I_{p} \tau}=\frac{e}{K I_{p} \tau}\left(\frac{I_{\text {mean }}-I_{D C}}{S_{\text {div }}}\right)^{2},
$$

where $I_{p}$ is the probe current, $e$ is the electron charge, and $\tau$ is the dwell time. The I $p$ was measured with a faraday cup. The $\eta_{\text {exp }}$ values were compared with the $\eta_{\text {ref }}$ reference data.

\section{Results}

Figure 5 shows the comparison of $\eta_{\exp }$ and $\eta_{\text {ref }}$ for (a) carbon, (b) silicon, (c) copper, and (d) gold at the landing energy between 0.2 and $1 \mathrm{keV}$. In all four materials, the $\eta_{\text {exp }}$ values follow the $\eta_{\text {ref }}$ fairly closely at the lower landing energies. However, there are a few data points that show a deviation from the reference data at the higher landing energy.

Figure 6 shows the $\eta_{\exp }$ of each material as a function of the landing energy. The $\eta_{\exp }$ values for $\mathrm{Cu}$ and $\mathrm{Au}$ tend to be higher at the landing energy of around $1 \mathrm{keV}$ and then tend to decrease as the landing energy becomes lower. The $\eta_{\exp }$ of $\mathrm{Au}$ is the highest at the landing energy of $1 \mathrm{keV}$, but it becomes the lowest of the four materials at the landing energy of $0.2 \mathrm{keV}$. The decrease in the $\eta_{\exp }$ of $\mathrm{Cu}$ with beam energy is more gradual than that of $\mathrm{Au}$. In contrast to $\mathrm{Cu}$ and $\mathrm{Au}$, the $\eta_{\exp }$ of $\mathrm{C}$ and $\mathrm{Si}$ tend to be lower at the higher landing energy; these values tend to increase slightly when the landing energy becomes lower. The $\eta_{\text {exp }}$ of Si is higher than that of $\mathrm{C}$ at the landing energy between 0.2 to $1 \mathrm{keV}$, and it is the highest of the four materials at the landing energy of $0.2 \mathrm{keV}$. The $\eta_{\exp }$ of $\mathrm{C}$ is the lowest at the landing energy of $1 \mathrm{keV}$, but it becomes higher than that of $\mathrm{Cu}$ and $\mathrm{Au}$ at the landing energy of $0.2 \mathrm{keV}$.

To confirm these effects with actual imaging, a test specimen was used consisting of a piece of bare $\mathrm{Si}$ substrate next to another piece of $\mathrm{Si}$ substrate with a $50 \mathrm{~nm}$ thick film of $\mathrm{Au}$ deposited on it. Figure 7 shows the BSE images taken at the landing energies of $1 \mathrm{keV}$ and $0.2 \mathrm{keV}$. At the landing energy of $1 \mathrm{keV}$, Au appears brighter than $\mathrm{Si}$, whereas at the landing energy of $0.2 \mathrm{keV}$ the contrast was reversed. These images correspond with the graphs shown in Figures 5 and 6. 


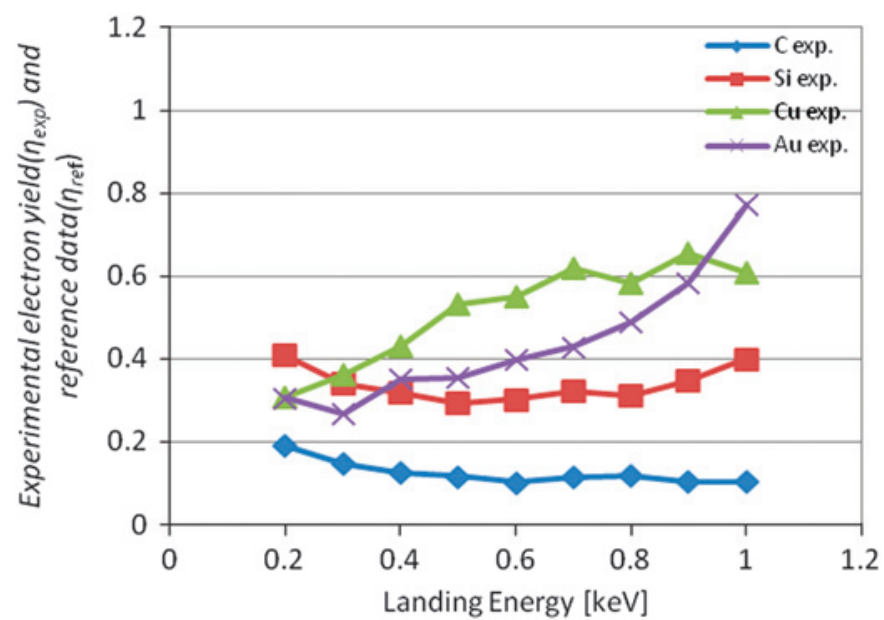

Figure 6: Experimental BSE yields $\left(\eta_{\text {exp }}\right)$ versus landing energy for carbon, silicon, copper, and gold.
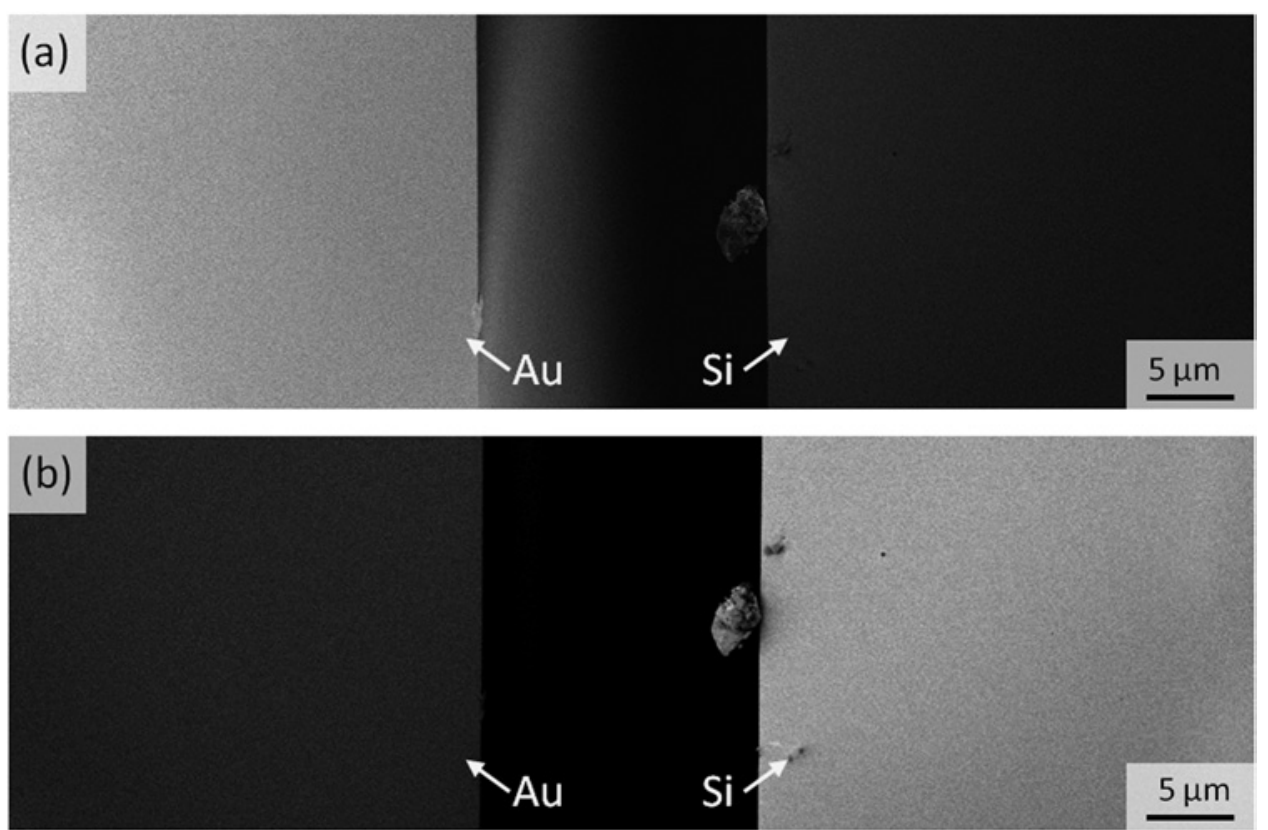

Figure 7: The reversal of BSE contrast between $\mathrm{Au}$ and $\mathrm{Si}$ acquired under the following conditions: accelerating voltage was (a) $1 \mathrm{kV}$, (b) $0.2 \mathrm{kV}$.

\section{Discussion}

The results shown in Figures 5 and 6 correspond reasonably well to the previous studies, except for a few data points. The relationship of $\eta_{\exp }$ versus $\mathrm{keV}$ for each material should correspond to the material contrast in the images. The material contrast at the landing energy around $1 \mathrm{keV}$ is proportional to atomic number, but it is not proportional at the lower landing energies. For the lower energies it becomes quite complicated. Gold is the brightest of the four materials at the landing energy of $1 \mathrm{keV}$. It becomes gradually darker at lower landing energies, and it is the darkest of the four materials at the landing energy of $0.3 \mathrm{keV}$. Copper is the second brightest of the four materials at the $1 \mathrm{keV}$ landing energy. It also becomes gradually darker at the lower landing energies, and it is the second darkest of the four materials at $0.2 \mathrm{keV}$. Silicon is the second darkest of the four materials at $1 \mathrm{keV}$. It becomes slightly brighter at the lowest landing energies and is the brightest of the four materials at $0.2 \mathrm{keV}$. Carbon is the darkest of the four materials at $1 \mathrm{keV}$. It becomes slightly brighter at the lower landing energies; it is the second brightest of the four materials at the landing energy of $0.2 \mathrm{keV}$. These tendencies of reversal have been explained theoretically by the much stronger deviations of elastic Mott cross sections from Rutherford cross sections at ULE [4]. This effect has been shown experimentally; at very low primary electron energies the BSE yields of heavier materials tend to decrease, and the BSE yields of lighter materials tend to increase [5-6].

There are several possible causes of the deviations of the experimental values from the reference data (Figure 5). One may be due to the difference in the sample state because the BSE yield is sensitively affected by factors such as cleanliness and flatness. There might be a little bit of contamination on the sample surface due to a hydrocarbon film (re)deposited by the electron beam, even though the vacuum in the chamber was very good, which is on the order of $10^{-5} \mathrm{~Pa}$, and the samples were carefully handled. In addition, instrumental factors such as the acceptance capabilities of the detector could affect the results. The complex phenomena occurring inside the chamber such as the generation of SE3 could affect the signal intensity as well.

\section{Conclusion}

The material contrast at the landing energy higher at $1 \mathrm{keV}$ is proportional to atomic number as expected, but it is not proportional to the atomic number at the landing energy of $0.2 \mathrm{keV}$. At the latter landing energy, $\mathrm{C}$ and $\mathrm{Si}$ were brighter than $\mathrm{Cu}$ and $\mathrm{Au}$. The $\eta_{\exp }$ values nearly correspond to the $\eta_{\text {ref, }}$, below about $0.5 \mathrm{keV}$, but there are a few experimental data points near $1 \mathrm{keV}$ that are higher than the $\eta_{\text {ref }}$ by a factor of two. Possible causes of the deviation include differences in sample state, contamination, the acceptance capabilities of the detector, and/or the complex phenomena occurring inside the specimen chamber.

\section{References}

[1] I Müllerová, Scanning 23 (2001) 379-94.

[2] D Joy, A Database of Electron-Solid Interactions Revision \# 12-1 (2012).

[3] D C Joy et al., Scanning 18 (1996) 533-38.

[4] L Reimer, Scanning Electron Microscopy, 2nd ed., Springer Science \& Business Media Verlag Berlin Heidelberg, New York, 1998.

[5] I Müllerová, Scanning 26 (2004) 18-24.

[6] MM El Gomati et al., Scanning 30 (2008) 2-15. 


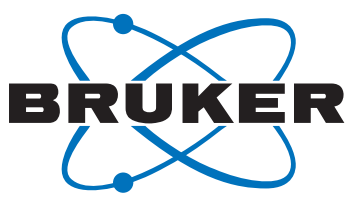

\section{Techniques - 1 Workflow.}

ESPRIT 2, the

only software

which combines

4 microanalysis methods.
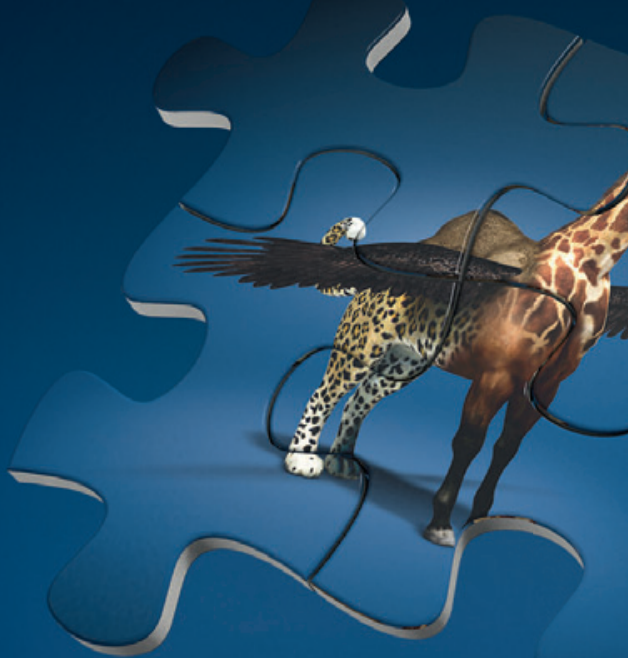

(1)
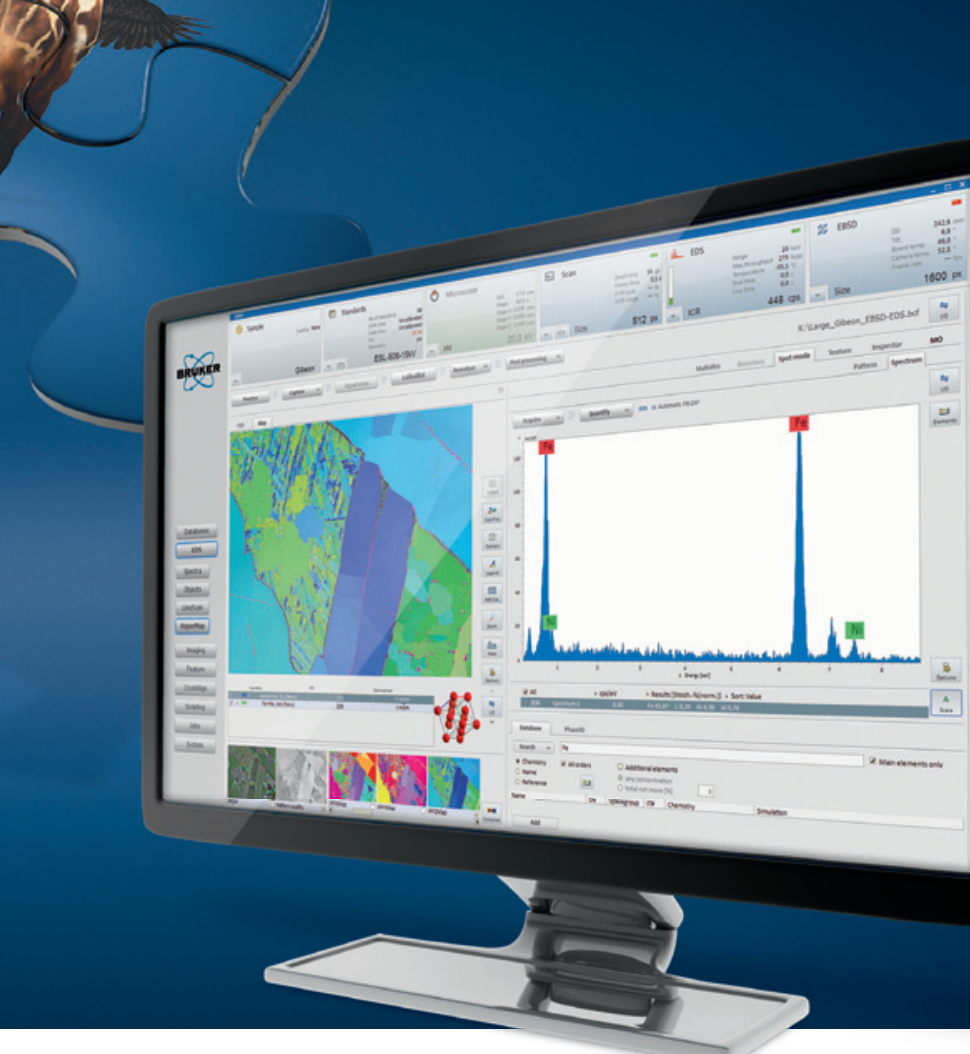

- Comprehensive management of analysis and results from EDS, WDS, EBSD and Micro-XRF with one software

- Complementary techniques provide you the most accurate and reliable results

- Zeta factor quantification for characterization of thin layers

Someone has to be first.

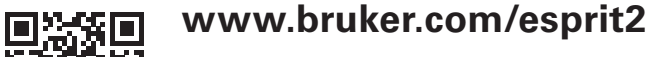

1.

wirit

口ifin

Innovation with Integrity 